\title{
Correction to: Data fusion of in situ geophysical and geotechnical information for levee characterization
}

\author{
T. Dezert $^{1,2}$ (D) S. Palma Lopes ${ }^{1} \cdot$ Y. Fargier ${ }^{2,3} \cdot$ L. Saussaye $^{2} \cdot$ P. Côte ${ }^{1}$
}

Published online: 28 April 2021

(C) Springer-Verlag GmbH Germany, part of Springer Nature 2021

Correction to: Bulletin of Engineering Geology and the Environment (2021) https://doi.org/10.1007/s10064-021-02225-2

Originally, the Eqs. 9, 10,12 and 13 were wrongly published in the online version. This has been correctly presented here. The original article has been corrected.

Equation 9

$$
m_{1,2, \ldots, s}(X)=\sum_{\substack{X_{1}, \ldots, X_{s} \in 2^{\Theta} \\ X_{1} \cap \ldots \cap X_{s}=X}} \prod_{j=1}^{s} m_{j}\left(X_{j}\right)
$$

Equation 10

$$
m_{1,2, \ldots, s}(\varnothing)=\sum_{\substack{X_{1}, \ldots, X_{s} \in 2^{\Theta} \\ X_{1} \cap \ldots \cap X_{s}=\varnothing}} \prod_{j=1}^{s} m_{j}\left(X_{j}\right)
$$

Equation 12

$$
m_{1,2, \ldots, S}^{D S}(X)=\frac{1}{1-m_{1,2, \ldots, S}(\varnothing)} \sum_{\substack{X_{1}, \ldots, X_{s} \in 2^{\ominus} \\ X_{1} \cap \ldots \cap X_{s}=X}} \prod_{j=1}^{s} m_{j}\left(X_{j}\right)
$$

Equation 13

$$
\begin{aligned}
m_{1,2, \ldots, S}^{P C R 6}(X)= & m_{1,2, \ldots, s}(X)+\sum_{k=1}^{s-1} \sum_{\begin{array}{c}
X_{i_{1}, X_{i}}, \ldots, X_{i_{k} \in 2^{\ominus} \backslash X}\left(\cap_{j=1}^{k} X_{i_{j}}\right) n X=\varnothing \\
\left(i_{1}, i_{2}, \ldots, i_{k}\right) \in P^{s}(\{1, \ldots, s\})
\end{array}}\left[m_{i_{1}}(X)+m_{i_{2}}(X)+\ldots+m_{i_{k}}(X)\right] \\
& \cdot \frac{m_{i_{1}}(X) \ldots m_{i_{k}}(X) m_{i_{k+1}}\left(X_{i_{k+1}}\right) \ldots m_{i_{1}}\left(X_{i_{s}}\right)}{m_{i_{1}}(X)+\ldots+m_{i_{k}}(X)+m_{i_{k+1}}\left(X_{i_{k+1}}\right)+\ldots+m_{i_{s}}\left(X_{i_{s}}\right)}
\end{aligned}
$$

The online version of the original article can be found at https://doi.org/10. 1007/s10064-021-02225-2

T. Dezert

theo.dezert@univ-eiffel.fr

1 Gustave Eiffel University, GERS, GeoEND,

F-44344 Bouguenais, France
2 Cerema Direction territoriale Normandie-Centre, F-41000 Blois, France

3 Gustave Eiffel University, GERS, RRO, F-69675 Bron, France 